\title{
EL ESTUDIO DE CASO Y SUS IMPLICACIONES METODOLOGÍCAS EN LA INVESTIGACIÓN: CASO DEL GOBIERNO LOCAL MEXICANO
}

\section{STUDY CASE AND METHODOLOGICAL INVOLMENTS IN RESEARCH: MEXICAN OF LOCAL GOVERNMENTCASE}

\author{
Vazquez Arellano Luis Ernesto ${ }^{1}$, Ramos García Jesús Manuel ${ }^{1}$ \\ 1 Universidad Autónoma Metropolitana - Azcapotzalco, Departamento de Administración, México, e- \\ mail: le_va787@yahoo.com.mx, jmaraga1@hotmail.com
}

\author{
ART ICLE IN F O \\ Article history: \\ Received 2020-01-15 \\ Accepted 2020-04-10 \\ Available online 2020-04-10
}

Palabras-clave: Metodologia. Gobierno local. Estudio de caso. Toma de decisiones.

Keywords: Methodology. Local government. Case of study. Make decisions.

RESUMEN. La cuestión metodológica es sin duda primordial para toda investigación, por muy simple o compleja que sea, toda vez que define de manera concreta no sólo la forma de conocer la realidad sino también los resultados esperados de manera fiable, viable y con la validez requerida; por lo que en este trabajo es el eje y objetivo principal, recalcar la importancia de definir la metodologia adecuada, a través de la cual hemos de dar cuenta de su importancia en la consecución de los objetivos planteados en la investigación llevada a cabo, relativa a la toma de decisiones en una instancia de gobierno local mexicano. En este sentido, es a través del estudio de caso que permitió vislumbrar la implicación metodológica, mostrando que su diseño y aplicación permite llegar a resultados confiables sobre una realidade; en particular de una institución local que se constextualiza dentro del ámbito metropolitano, realidade que está determinada por relaciones multidimensionales. Se observó que la influencia de la forma de organización adquirida por los actores y su entorno interno generan formas de trabajo más o menos complejas, aunadas a las externas, que conforman redes de interdependencia entre sí, como las relacionadas a las intermunicipales. Esta organización tuvo gran auge en las funciones de casi toda la administración del gobierno local, visión que se pudo interpretar con la aplicación de la metodología selecionada.

\begin{abstract}
Methodologyl is essential for any research, independently if is simple or complex, whenever it defines in a concrete way not only the way of knowing reality but also the expected results are reliable, viable way and with the required validity. In this document, it is the axis and main objective, to emphasize the importance of defining the appropriate methodology, through we must realize its importance in achieving the objectives set in the research carried out, related to decision making in an instance of Mexican local government. In this sense, it is through the case study that the methodological implication is glimpsed, showing that its design allows to reach reliable results on a particular reality in a local institution, contextualized in a metropolitan area, reality that is established by multidimensional relationships. We verify the influence of organization form acquired by the actors and their internal environment produce more or less complex forms of work, attached to the doors, which create networks of interdependence with each other, such as those related to inter-municipal ones. This organization had a great boom in the functions of almost the entire local government administration, a vision that we interpreted with the application of the selected methodology.
\end{abstract}




\section{Introducción}

La base fundamental de este trabajo se encuentra en la publicación de las Actas del CIAIQ 2019, cuya esencia es la investigación y las implicaciones del caso de estúdio en una instancia de gobierno local. De esta manera, se puede señalar que la principal actividad que requiere todo investigador es el cuestionamiento o duda sobre una realidad en particular; sin embargo, para que se lleve a cabo la indagación de la realidad que se desea conocer o la particularidad de un fenómeno, la elección de la metodología que permita alcanzar los objetivos planteados es trascendental, toda vez que implica la accesibilidad al conocimiento o restricciones para lograr los resultados esperados, por tanto, como principal objetivo del presente trabajo es demostrar que la elección de la metodologia es central, dado que tiene implicaciones a lo largo de la investigación a realizar. Desde la elección de la metodologia se pueden percibir profundas implicaciones que guiarán una investigación, ya sea de corte cualitativa, cuantitativa o mixta; lo que conlleva a abordar al sujeto de estudio desde una u otra perspectiva teórica electa.

El método, con sus limitaciones y ventajas, coadyuvan a un acercamiento de una realidad que se pretende conocer, tomando en consideración que sólo es un cohorte de ésta y que diversas disciplinas pueden dar cuenta de ella bajo otros enfoques o teorías. Al hacer uso del método de estudio de caso aportó elementos que permitieron concluir sobre la particularidad y singularidad de un sujeto de estudio y con ello explicar un fenómeno de nuestro interés.

En la investigación llevada a cabo en este trabajo se da a conocer el tópico y fenómeno sobre la toma de decisiones en torno al tema urbano dentro de una dependendencia de gobierno local en México. Lo anterior nos permite señalar que las decisiones que toman los actores en el contexto metropolitano son complejas, en tanto que la multiplicidad de dimensiones, interacciones e interrelaciones es amplia; observando un entretejimiento de diversas decisiones que no son aisladas e independientes.

De esta manera, el trabajo se estructura, primero, una parte donde se reflexiona en torno al proceso de investigación, dando cabida al método utilizado. Posteriormente se da cuenta de la aplicabilidad del método y los resultados principales, retomando aquellos más relevantes y, finalmente, emitir reflexiones, de acuerdo a la relación metodologíainvestigación-caso de estúdio; com la finalidade de dar cuenta de esta relacion instrínseca em los procesos de investigación que usan esta metodologia. 


\section{Cuestiones metodológicas en la investigación: Algunas reflexiones}

Entre los diversos retos que enfrenta todo investigador no sólo es generar y aportar al conocimiento, sino también garantizar que éste corresponda y de prueba de una realidad desde el enfoque o perspectiva cualquiera que sea. En este sentido, una de las bases recae en el uso de la metodología que permita allegarse del conocimiento de esa realidad y que de validez y confiabilidad al proceso de investigación, en cuyo caso puede ser aprobada por otros investigadores u otras instancias a través de los diversos instrumentos.

Sin embargo, esta relación entre investigación y metodología no es única, pues Según Leal (2017), existe otra parte fundamental en todo el processo, la parte argumentativa, cuyo enlazamiento y entretejimiento de ideas van sustentando y dando coherencia a un cuerpo total de expresión que se plasma, usualmente, en forma escrita, a lo que, comúnmente se le denomina como el informe de investigación; cabe aclarar que lo anterior no significa el cumplimiento de esta premisa, pues muchos de los documentos llegan a ser descriptivos o narrativos, em el mejor de los casos. En los mismos argumentos de Leal (2017), subraya la discrepancia entre lo metodológico (que alude a una familia que incluye la teoría, el marco teórico, modelo, hipótesis, técnicas de investigación, entre otros) y la cuestión argumentativa (incluye términos como argumento, argumentación, premisa, conclusión, falacia); y propone que no debería de haber un desencuentro, muy al contrario, la complementariedad como eje que reajusta el proceso de investigación a ser más preciso, conciso y confiable.

Además, es de considerar que no se puede desvincular u omitir al proceso de investigación a la cuestión argumentativa, pues, de acuerdo a la Universidad de las Américas de Puebla (DLAP) (2015) es a través de éste que se sustenta una idea o investigación, dando pruebas, ofreciendo razones, inferencias o argumentos a ésta; es decir, con evidencias. En toda investigación, el argumento está presente en todo el cuerpo del documento escrito. Aunque no es el propósito de este trabajo ahondar en la cuestión argumentativa, creemos conveniente precisar su importancia.

Otro de los aspectos que definen el rumbo de una investigación es la definición de la metodologia, que se adecue y que que permita aprehender la realidad pretendida y, por lo tanto, generar conocimiento científico. Pero, en ideas de Samaja (2005) la investigación no se aprende en los cursos o diplomados de manera per se, sino que éste se da en la constante praxis, con la aplicación y autocrítica del quehacer; lo anterior implica que el investigador adquiera experiencia y habilidades para conocer qué camino tomar en el proceso científico de investigación de acuerdo al objeto, objetivo, etc., de estudio.

La investigación social y aplicada son las dos primeras formas en que se conoce como la primera forma de investigación, a las cuales se les relaciona lo cualitativo y cuantitativo, 
respectivamente. En este caso, la cualitativa es la que se aborda; que de acuerdo a Hernández et. al. (2010) sostiene que sus características son: explora los fenómenos en profundidad; se conduce básicamente en ambientes naturales; los significados se extraen de los datos y, no se fundamenta, principalmente, en la estadística.

La investigación social es compleja en tanto que hay diversas epistemologías mediante las cuales se puede abordar a los individuos en sociedad y en cualquier forma de organización. Para el caso que nos ocupa, la naturaleza de los actores en las organizaciones gubernamentales y sus interacciones por relaciones interorganizacionales y su propia construcción individual son centrales para conocer al realidd en torno a las decisiones a través de la aplicación del estúdio de caso, por lo que resulta pertinente para este trabajo.

\subsection{La investigación a través del estudio de caso}

Es importante destacar el enfoque cualitativo; que de acuerdo a Hernández et. al. (2010), tiene las siguientes características:

1. Explora los fenómenos en profundidad

2. Se conduce básicamente en ambientes naturales

3. Los significados se extraen de los datos

4. No se fundamenta, principalmente, en la estadística

En tales características, se requiere buscar la forma en cómo obtener información, primero, acorde al fenómeno estudiado, y segundo, el cómo allegarse de la información precisa y clave de las fuentes de información. La subjetividade, se vuelve central em este ejercicio de investigación.

Así, la subjetividad en ciencias sociales alude, esencialmente, a fenómenos intangibles ya sean individuales o colectivos. El estudio de caso, justamente, nos permite abordar esta complejidad, que requiere de técnicas e instrumentos de investigación acordes para aprehender esa realidad y construirla, de tal manera que la comprensión del fenómeno sea lo más objetiva posible.

De acuerdo a Soto (2013) el método de caso de estúdio hace referncia a la recolección y selección de información acerca de la biografía, personalidad, intenciones y valores que pueden ser de cualquier persona, institución, programa, responsabilidad, población u organización. La validez y carácter probativo depende de su realidad, de su autenticidad y no de su frecuencia o de su representatividad con respecto a un promedio estadístico. De igual manera, señala que lo importante del caso de estudio es el análisis de una situación auténtica en su complejidad real, de alguna manera refiere la comprensión de las relaciones de función entre hechos y acontecimiento unos de otros, lo cual se puede apreciar en la siguiente definición: 
"El estudio de caso suele considerarse como instancia de un fenómeno, como una parte de un amplio grupo de instancias paralelas (...) un dato que describe cualquier fase o el proceso de la vida entera de una entidad en sus diversas interrelaciones dentro de su escenario cultural -ya sea que esa unidad sea una persona, una familia, un grupo social, una institución social, una comunidad o nación. ... dependiendo de las relaciones entre factores dentro de la unidad total" (YOUNG en Arzaluz 05, p. 133, citado en DÍAZ et. al., 2011, p. 3).

La particularidad de los estudios de caso es que se centran en los niveles micro o local sin, necesariamente, mantenerse aislado a la totalidad; es decir, hablamos de uma integralidad que permita comprender el todo. Se parte de una concepción humanista y de enfoques sociocríticos donde se reconoce la complejidad (SOTO, 2013). Asimismo, refiere cuatro tipos de abordar al estudio de caso: de una forma particularista (se centran en una situación, evento, programa o fenómeno en particular), descriptiva (es básicamente una descripción rica y densa del fenómeno objeto de estudio), heurística (dan lugar al descubrimiento de nuevos significados) e inductiva (se descubren nuevas relaciones y conceptos). En nuestro caso, nos centramos en un proceso, que es la toma de decisiones, por lo que a pesar de ser particular, conlleva múltiples relaciones que se integram y son, em muchos casos, interdependientes.

Para abordar el estudio de caso como método, señala Díaz et al. (2011) el investigador se somete a: un protocolo de investigación, contextualización del problema y describir la unidad de análisis y determinar el método de análisis, organizar los datos obtenido y representarlos de manera que se observen claramente los elementos y relaciones entre ellos y la unidad de análisis, es decir una sincronía.

Un aspecto que de los estudios de caso que resulta importante en esta investigación es que con este método se busca a detalle la interacción entre los contextos (STAKE, 1999); la particularidad y complejidad de un caso singular. El investigador cualitativo busca los detalles, y tomar en cuenta lo holístico, es decir, el todo. Y como señalara Stott y Ramil (2014) bajo este método se puede contribuir a cambios significativos en los ámbitos de la política y la práctica.

\section{Metodología aplicada}

La aplicacación del estudio de caso como método hace posible comprender una realidad particularizada en cualquier tipo de organización; realidade que implica abordar elementos dirigidos para compreender el fenómeno en cuestión. El caso que nos ocupa es la instancia gubernamental que se encarga de todo lo relacionado al tema urbano, siendo un municipio de corte metropolitano y capital de estado. De lo cual se dará cuenta en los resultados principales. 
Para llevar a cabo la aplicación del método de estúdio de caso, la pregunta de investigación que fue el hilo conductor para la comprensión y construcción de una realidad fueron: ¿Cómo se dan los procesos decisorios en las organizaciones gubernamentales locales dentro de las estructuras institucionales? ¿Cómo son las interrelaciones tanto formales como informales entre los diferentes actores que se involucran en los procesos de decisión en las organizaciones gubernamentales? Usando metodología cualitativa y, siendo el método de estudio de caso, el que nos permite comprender esta realidad. Por lo tanto, tomando en cuenta el caso de estudio; el diseño metodológico se compone de la siguiente manera:

- Sujeto de estudio: organización gubernamental local

- Objeto de estudio: la toma de decisiones (política pública)

- Se utiliza el enfoque cualitativo

- Por la naturaleza del fenómeno, el paradigma interpretivo es fundamental para conocer esta realidad; así como la revisión y análisis documental (normas, internet, información de archivos de la organización).

- La estrategia metodológica sugiere el estudio de caso, toda vez que no se pretenden abordar más de dos y mucho menos llegar a generalizaciones, sino más bien, dar cuenta de la particularidad del fenómeno en la organización.

- La perspectiva analítica se ubica en la de los estudios organizacionales

- Se concibe al proceso de construcción moderada de toma de decisiones como la unidad en la que se enfocará el análisis, cuyos elementos se ligan a un contexto de la organización, del fenómeno y la forma en cómo se estructura la organización para facilitar o limitar la toma de decisiones.

- Técnica de investigación: entrevista semiestructurada. Que de acuerdo a Brewerton y Millward (2001: 69-70) se basan en una guía de asuntos o preguntas adicionales para precisar conceptos u obtener mayor información sobre temas deseados (no todas la preguntas están determinadas).

El muestreo utilizado en la investigación fue mediante muestras dirigidas, esencialmente aquellas a expertos. Esto significa que informantes clave otorgan información pertinente para comprender el problema o realidad de la toma de decisiones, esto es, que son de relevancia los actores en mandos medios y altos, puesto que perciben y son parte de las decisiones y conocen la forma en cómo se llevan a cabo; por lo que se considera que son los principales partícipes y fomentadores o limitadores de la participación dentro y fuera de la organización. La selección de ellos se llevó a cabo considerando su influencia en la toma de decisiones, en la información privilegiada que poseían y de acuerdo al organigrama analizado; dando un total de 6 informantes, de acuerdo a la estructura de la organización. 
Las dimensiones o categorías de análisis que permitieron conocer la toma de decisiones fueron: coordinación, horizontalidad, lazos de confianza, relación con la sociedad y manejo de conflicto, lo anterior, considerando a Torres (2015). El autor señala que el primero se refiere a cualquier tipo de instrumento que permita consensuar; el segundo a una menor jerarquización al interior de la organización; el tercero al acceso a la información y formas de comunicación de forma irrestrictiva; el cuarto a la forma en que fomenta la participación interna como externamente y el quinto a la búsqueda de un ambiente tanto social como laboral sano.

\section{Principales resultados}

El lapso que duro la investigación fue por 4 años, aludiendo a un periodo de gobierno municipal específico (2013-2015). Se inició con el planteamiento del problema, la pregunta de investigación que nos guiaría [con la pregunta de investigación planteada, permitió un mayor análisis, que enriqueció la interpretación de la información] y la justificación, para dar cabida a la literatura o desarrollo del marco teórico conceptual, posteriormente el diseño metodológico y la determinación de los instrumentos de recolección de la información (entrevista semiestructurada, cuaderno de notas, oservación no participante), para su posterior aplicación; cuya duración fue de casi dos años, aplicándola en las visitas a la organización.

El acercamiento a la organización fue fundamental, siendo a través de contactos dentro de la administración y visitas directas con los actores de nivel superior, lo que permitió lograr acceder no sólo a los informantes, sino también a la información documental y a la observación directa. A partir de ahí, y con base en el instrumento de recolección de datos elaborado, se realizaron 6 entrevistas a informantes clave, las cuales fueron grabadas en audio con tiempos diversos con cada uno, para después transcribirlas en el programa word y posteriormente llevar a cabo el análisis de la información a partir de secciones definidas que permitieron analizar las categorías de análisis con base en las respuestas y de acuerdo a las dimensiones planteadas, haciendo uso del interpretativismo. Cabe aclarar que las entrevistas requirieron de varias citas, ajustándose a la disponibilidad de los informantes.

El proceso de investigación que nos permitió llevar a cabo la investigación se aprecia en la figura 1. 


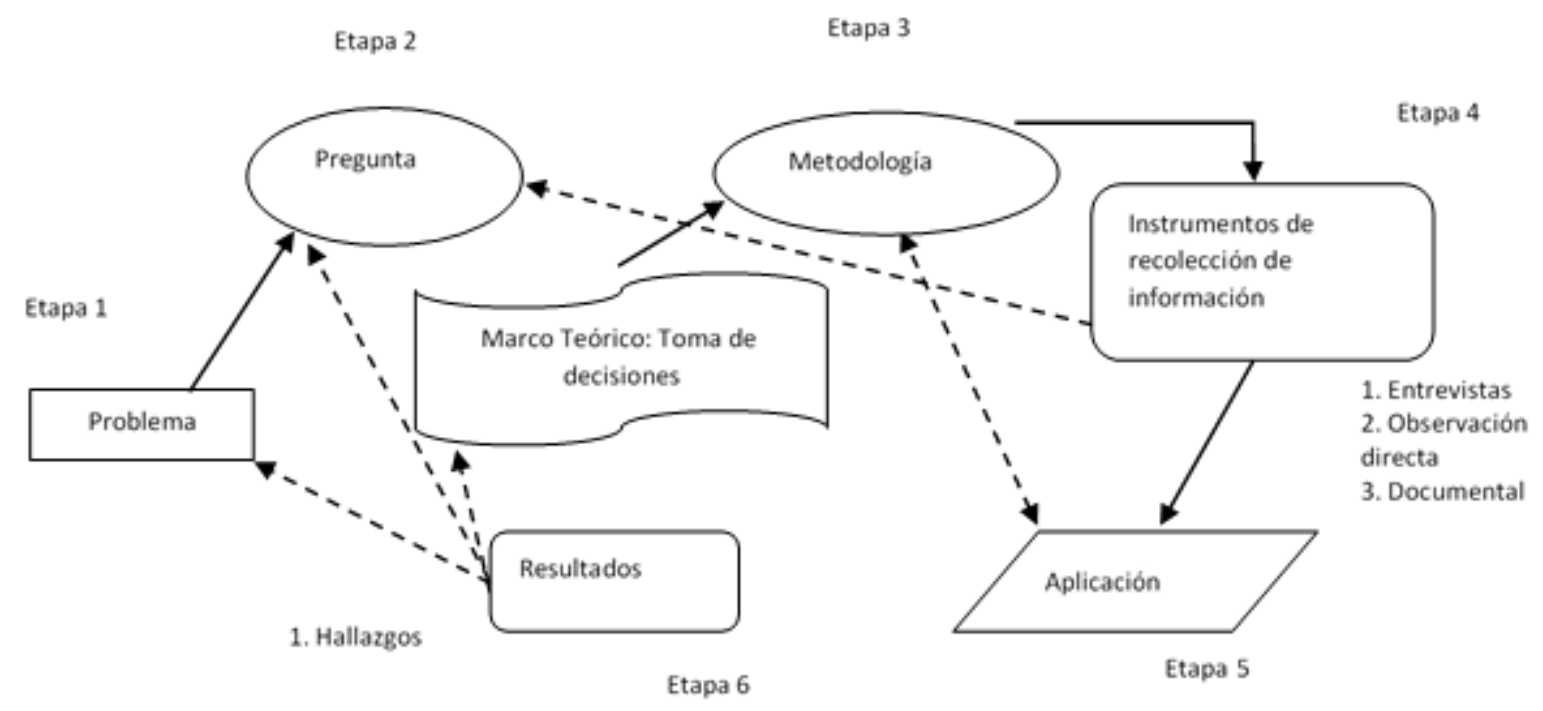

Figura 1 - Etapas y relaciones del proceso de investigación

Fuente: Elaboración propia (2013)

\subsection{Elementos teórico-conceptuales}

Los gobiernos municipales como parte de una estructura amplia dentro del denominado federalismo, se constituyen como el principal ente que tiene gran interacción con el ciudadanos; son considerados, en términos de Arellano, Cabrero y Del Castillo (2000: 11) como “...espacios creados en la dinámica de estructuras heterogéneas. ...no son rígidas y lineales sino que se desarrollan a través de esquemas interpretativos y de acciones estratégicas contingentes de un sinnúmero de actores al amparo de reglas, recursos y formas organizativas". Asimismo, señalan que el gobierno también es un conjunto de organizaciones dentro de un espacio institucionalizado. El conjunto de leyes, normas, costumbres, espacios creados desde el régimen y el sistema político, son elementos constitutivos primarios de cualquiera organización gubernamental.

El município surgió como forma de organización representativa de la sociedad local, que como señala Morelli (2007) su surgimiento se debió a cuestiones territoriales, a una fragmentación de las monarquías y como forma de representación local, los móviles de creación eran de carácter urbano dejando de lado aquellas localidades rurales.

Así, las organizaciones gubernamentales son duales, se deben a una doble lógica: nacen predeterminadas, en sus fines y en los mecanismos generales de acción y obtención de resultados. A la vez continúan siendo organizaciones por sí misma, con su vida interna en su contexto particular. Esto se refleja en la variedad de formas de organización de estados y municipios, creando, desapareciendo o cambiando estructuras organizacionales de acuerdo 
a la visión del actor. Sin embargo de acuerdo a Hernández y Osegueda (2009) éstos son arenas de conflictos ya que la distribución del poder es injusta.

Por lo tanto, muchas de las decisiones que se toman en este ámbito, repercuten localmente e incluso escalan a otros. En este tenor, se retoma el concepto de Simon (1988: 10) que lo define como el "proceso mediante el cual se hace una selección de entre una variedad de alternativas sobre una situación o asunto, llegando a una decisión sobre una de ellas". Anticiparse al futuro es una de las aspiraciones de las decisiones de los seres humanos, tratando de evitar, en lo más posible la incertidumbre.

La complejidad del concepto implica tomar alguno de ellos, por lo que se considera con mayor apego a la investigación el de Corzo (2011:3) que señala indica que "constituyen el resultado de un proceso reflexivo y consciente, basado en información, que se inicia con una situación de insatisfacción (problema) y búsqueda de soluciones, mediante el análisis de por lo menos dos alternativas de estrategias futuras, mutuamente excluyentes...", decisiones inmersas en un contexto socioeconómico-territorial que alberga múltiples actores. En términos de Lascoumes y Galés (2014) hay dos formas de decisión, aquellas decisiones que provienen desde abajo (bottom up) y por el otro desde arriba (top down).

Con la delimitación del concepto basado en las decisiones racionalmente limitadas, se puede señalar que pueden manifestarse múltiples-racionalidades, y de acuerdo a Lascoumes y Galés (2014) estas diversas racionalidades están en competência, ya sean de carácter colectivo o individual.

\subsection{El sujeto de estúdio}

La organización en la que se llevó a cabo la investigación se encuentra en el Estado de México, Estado que se ubica en la parte centro del país. Geopolíticamente, el Estado de México está dividido en 125 municipios, siendo Toluca la sede de la Capital de la entidad.

Funcionalmente, la Región del Valle de Toluca, que se integra por 22 municipios metropolitanos, tiene una ubicación estratégica en el centro del país, lo que le permite aprovechar su relación con la megalópolis que se forma alrededor de la Zona Metropolitana del Valle de México, articulando su desarrollo al Corredor Golfo - Pacífico y al denominado eje del Tratado del Libre Comercio con América del Norte y, en consecuencia, le permite aumentar su competitividad respecto a las otras zonas metropolitanas de la región Centro del País, por lo que los Proyectos Estratégicos del Estado tienden a consolidar esta articulación. La industrialización del país en los años sesenta y setenta, marcó la transformación de Toluca de ciudad media, con función administrativa estatal, a convertirse en el centro de una metrópoli de influencia regional y nacional. Se estima que la población de la Zona Metropolitana del Valle de Toluca para el año 2020 será de 1 millón 858 mil habitantes. Las densidades de la Región se cuadruplicaron entre los años 1960 y 2000, al incrementarse de 164 a 649 habs. 
/km2., y en los 9 municipios de la Zona Metropolitana Conurbada del Valle de Toluca de 179 a 770 habs. $/ \mathrm{km} 2$.

La instancia encargada del tema urbano es el Instituto Municipal de Planeación, cuyo antecedente fue la Dirección General de la Administración Urbana y Obras Públicas. De esta manera, la visión inicial era que la toma de decisiones se concentraba en lo absoluto en la segunda. Cuando se inició con el trabajo de campo, se descubrió que las decisiones habían sufrido transformaciones importantes. Tradicionalmente, las decisiones han obedecido a una alta jerarquización en la que los mandos medios y altos tienen en su poder las decisiones a discrecionalidad.

\subsection{Resultados de la investigación}

Primeramente, lo que arrojó la investigación com base en la apalicación metodológica, fue la existencia de una reestructuración organizativa loca, pasaando de uma Dirección General, a la creación de um instituto y a la disminución de funciones de la primera.

La reestructuración sufrida en la administración pública municipal y por ende en la política urbana, según los actores que dirigen las áreas, ha implicado que haya relaciones e interacciones, principalmente entre ambas (IMPLAN-Dirección de Administración Urbana y obras Públicas); sin embargo, ello no implica que ambas no fortalezcan relaciones con otras dependencias no sólo de la estructura organizacional del ayuntamiento, sino también con otras del gobierno estatal y del gobierno federal, habiendo coincidencias en algunas de estas interacciones. Lo anterior, inevitablemente implico ajustes em la toma de decisioes em torno al tema urbano; incluso, se podría indicar que las decisioes se fragmentaron; pero que no necesariamente se hacían de manera aislada; sino que implico crear mecanismos de coordinación y consenso para tomar decisiones. La reestructuración se muestra en la figura 2.

Asimismo, tenemos que, el Instituto Municipal de Planeación (IMPLAN) es un órgano desconcentrado de la administración pública municipal, cuyas funciones esenciales son: Planear, proyectar, diseñar y coordinar, orientar, asesorar, emitir la normatividad y elaborar los planes, programas, proyectos conceptuales y acciones, así como evaluar y coordinar con la Presidencia Municipal la iniciativas que contribuyan al diseño de políticas públicas para el ordenamiento del territorio de los asentamientos humanos. Junto a la Dirección de Administración Urbana y Obras Públicas se toman las decisiones sobre el tema. 


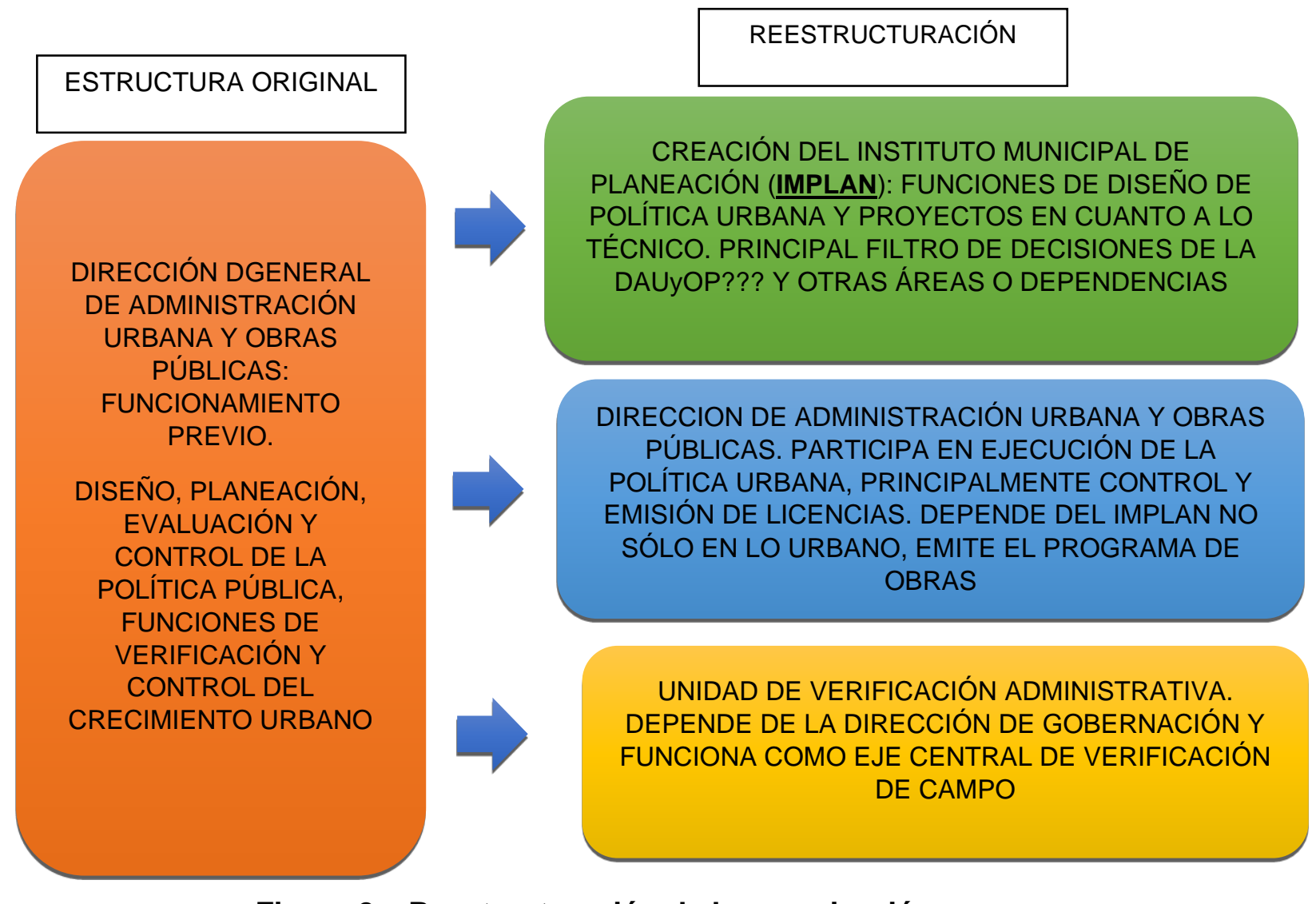

\section{Figura 2 - Reestructuración de la organización}

Fuente: Elaboración propia (2015)

Sin embargo, las funciones se transformaron en la que la parte pensante recaía en el IMPLAN y la operatividad o ejecución en la segunda, adicionalmente, Gobernación se encargaba del control y vigilancia de la actividad urbana a través de notificadores. Estas transformaciones constantes, como lo señala Arellano y Cabrero (2000), son parte de la vida de las organizaciones en la que las estructuras, las reglas y sistemas interpretativos son trascendentales.

Por otro lado, el IMPLAN surgió a partir de una forma de collage [en términos del institucionalismo sería uma espécie de mimetización organizativa], retomando aquellos aspectos de éxito de otras experiencias en el país, tales como el de Aguascalientes, León, San Luis Potosí y Puebla. Al adquirir esta forma organizativa, las decisiones se fragmentaron; sin embargo, a decir de los actores, fue mucho más fluido el tomar decisiones, sin concentrar todo el proceso en una sola instancia. El análisis de la problemática fue más asertivo, en la que los diversos actores de mandos medios y operativos participaban bajo sus diversos puntos de vista

Para dar vida al IMPLAN, los arreglos institucionales formales e informales creados permitieron un funcionamiento que apoyaba las decisiones de forma más eficaz, en la que los diversos actores, tanto internos como externos a la organización tuvieran cabida. Estos 
arreglos se observan en la figura 3. La cual señala no sólo los de carácter federal, sino también municipal, formando um sistema.

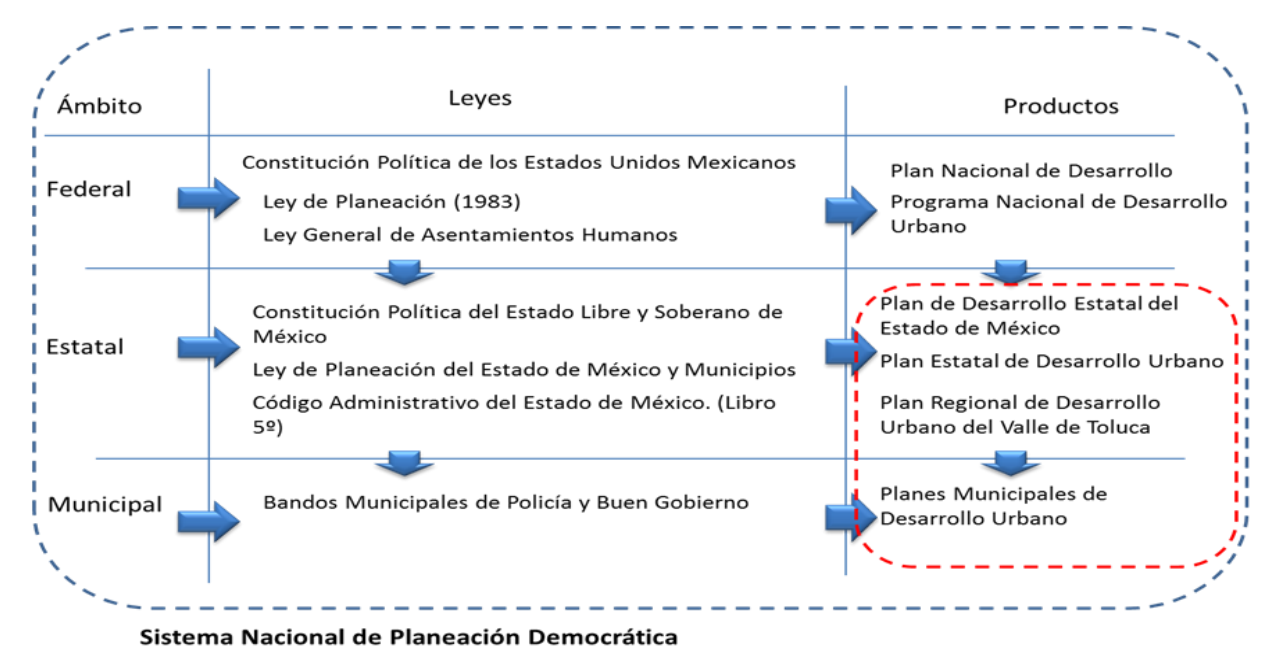

Figura 3 - Marco sobre principales arreglos institucionales y de actuación sobre los actores.

Fuente: Elaboración propia (2015)

Con las entrevistas llevadas a cabo, se reconoció la multiplicidad de interacciones e interrelaciones que los actores sostenían, tanto interna como externamente. En la toma de decisiones se hace a través de un cuerpo colegiado integrado por las subdirecciones y departamentos del instituto, en el cual se discuten los problemas y se llegan a acuerdos consensuados. En estos acuerdos se define cómo abordar el problema y si hay soluciones, se decide si hay cambios, lo anterior con la finalidad de llegar a soluciones o decisiones consensuadas entre los actores. El hecho de que los actores tuvieran profesión, facilitó que el diálogo fuera fluido. La atención de los problemas y la forma en que se agendaban respondía a consensos y la interacción con los sectores externos.

Sobre las dimensiones que explican el fenómeno de la toma de decisión se descubrió que la procuración de estas variables por parte de los actores. En el caso de la coordinación había convenios con Instituciones Educativas, Empresariales y Sociales e Intermunicipales (metropolitana); sin embargo, eran débiles en tanto la resistencia de otros actores (de otros municipios). Con respecto a la horizontalidad, se aprecian rasgos al respecto, pues aunado a los lazos de confianza, generaban un ambiente más horizontal; cuestión que no era percibida ni sentida por la mayoría de los actores, pues se observó que la secretaria del director poseía un gran poder.

Con respecto a la dimensión de la relación con el sector social y privado, se ha dado cabida a la apertura a los diversos actores no gubernamentales, como lo fue la participación 
en la actualización del Plan de Desarrollo Municipal, además de la apertura del directos a las demandas ciudadanas; las cuales se enlazan a la última dimensión, el manejo del conflicto, en cuyo caso, internamente había la apertura a opiniones críticas y conflictos entre pares, por el otro, a los conflictos externos e independientes a la organización, que por su naturaleza, debía tomar en cuenta.

Finalmente, reconociendo que las organizaciones se transforman, terminado el periodo de gobierno y llegando el entrante, a pesar de ser de la misma institución política, desapareció el IMPLAN y se denominó como Dirección de Administración Urbana y Movilidad Sustentable. Con la operación de la estrategia metodológica se muestra que fue pertinente, toda vez que permitió conocer la realidad de una organización de forma particularizada en un momento dado.

\section{Conclusiones}

Comprender una realidad a partir de una investigación requiere de seleccionar la metodología adecuada para lograr este objetivo; en la experiencia de este caso, se demuestra que los resultados dependen en gran medida de ello; aunque no se descarta que a lo largo, la flexibilidad metodológica debe estar en un proceso de aprendizaje. Se pudo observar que el sujeto de estudio se comprende a partir de elementos teórico-conceptuales que logran explicar un fenómeno. Las implicaciones de una metodología sobre la investigación conducen a estos resultados y su confiabilidad. La relevancia del uso del caso de estudio como método implica el conocimiento de realidades concretas en las que la identificación de problemáticas o áreas de oportunidad son oportunas. Este tipo de estudio dan cabida a nuevas investigaciones que bien pueden estar dentro de estudios comparativos en un país o respecto de otros.

Para el caso de estudio, conocer la forma en que se toman las decisiones en una instancia local implicó acercarse a una organización que se transforma continuamente, en la que la visión de los actores centrales influye en las formas organizativas. Para el periodo estudiado, las decisiones eran más analizadas, con actores profesionales y formas de trabajo más horizontales, en las que el ambiente externo influyó a través de los actores no gubernamentales. El posicionamiento del municipio implicó decisiones complejas; lo anterio se debe a diversos factores que entran en juego en esta "arena". Entre muchos otros, las cuestiones de poder, la heterogeneidad de actores en torno a la problemática urbana, las restricciones de estructuras jerárquicas hacia el ámbito local, al sistema de reglas y normas y los procesos dinámicos sociales, económicos y urbanos. 


\section{Referencias}

ARELLANO, David; CABRERO, Enrique; DEL CASTILLO, Arturo (Eds). Reformando al Gobierno. Una visión organizacional del cambio gubernamental. CIDE y Miguel Ángel Porrúa, México, 2000

AYUNTAMIENTO DE TOLUCA. Manual de la Dirección de Administración Urbana y Obras Públicas., México, 2014

AYUNTAMIENTO DE TOLUCA. Manual de Organización del Instituto Municipal de Planeación, Toluca, Estado de México, 2014

AYUNTAMIENTO DE TOLUCA. Manual de Procedimientos del IMPLAN, Toluca, Estado de México, 2014

H. AYUNTAMIENTO DE TOLUCA. "Plan de Desarrollo Urbano Municipal", Aprobado el 12/09/2014, Modificación. 2014

BREWERTON, Paul; MILLWARD, Lynne. Organizational Research Methods. A Guide for Students and Researchers. SAGE. 2001

CORZO, Abraham. Godelier y la racionalidad e irracionalidad en la economía. Magazine Cultura de Debate. 2010. Texto disponivel em: http://culturadedebate.blogspot.mx/2010/03/godelier-y-la-racionalidad-e.html, Acceso em: 27 de nov. 2016

DÍAZ, De Salas Sergio Alfaro; MENDOZA, Martínez Victor Manuel; PORRAS, Morales Cecilia Margarita (2011). Una guía para la elaboración de estudios de caso. Texto disponivel em: http://www.razonypalabra.org.mx/N/N75/varia_75/01_Diaz_V75.pdf. Acceso em: 20 de dic. 2015

GOBIERNO DEL ESTADO DE MÉXICO (2008) Plan Estatal de desarrollo Urbano. Aprobado el 19/05/2008

GOBIERNO DEL ESTADO DE MÉXICO (2008) Plan Regional de Desarrollo Urbano del Valle de Toluca. Aprobado el 9 de agosto de 2005 en Gaceta de Gobierno GOBIERNO DEL ESTADO DE MÉXICO. Ley de Planeación del Estado de México y Municipios, Texto disponível em: http://www.edomex.gob.mx/legistelfon/doc/pdf/ley/vig/leyvig087.pdf. Acceso em:02 de dic. 2011.

GOBIERNO DE LA REPÚBLICA. Ley General de Planeación, Gobierno de México, 1930 AYUNTAMIENTO DE TOLUCA. Manual General de la Administración Pública Municipal de Toluca 2013-2015. Ayuntamiento de Toluca, México.

LASCOUMES, Pierre; GALÈS, Patrick Le. Sociología de la acción pública. México, El Colegio de México, 2014 
LEAL, Carretero Fernando. ¿Qué función cumple la argumentación en la metodología de la investigación en ciencias sociales? Espiral (Guadalajara), vol 24, no. 70, p 9-49. Disponivel em: http://www.scielo.org.mx/scielo.php?script=sci arttext\&pid=S166505652017000300009. Acceso em: 08 de enero.2017

HERNÁNDEZ, Abiram; OSEGUEDA, Alfonso. Manual de gobierno municipal en México. Preguntas y respuestas sobre el Gobierno Municipal. CESEM (Centro de Servicios Municipales Roberto Jara A. C., Rosa Luxemburg-Stuffing. 2009

HERNÁNDEZ, Sampieri Roberto, et. al Metodología de la investigación, MacGrawHill, México, Bogotá, Buenos Aires, Caracas, Guatemala, Madrid, Nueva York, San Juan, Santiago, Sao Paulo, Auckland, Londres, Milan, Montreal, Nueva Delhi, San Francisco, Singapur, Saint Louis, Sidney, Toronto, Quinta Edición. 2010

MORELLI, Federica.. Orígenes y valores del municipalismo Iberoamericano, Auricaria, año /vol. 9, número 018, Universidad de Sevilla, España pp. 116-129. 2007

SAMAJA, Juan Alfonso. Epistemología y metodología: elementos para una teoría de investigación científica. Ediorial Universitaria de Buenos Aires, Argentina. 2004

SIMON, Herbet. The New Science of Management Decision. Harper and Row, New York. 1960

SIMON, Herbert, El comportamiento administrativo, Estudio de los procesos decisorios en la organización administrativa, Aguilar, Buenos Aires. 1988

SOTO, Ramírez Ricardo. Método: estudio de casos. Texto disponível em:http://www.paginaspersonales.unam.mx/files/981/estudio_de_caso.pdf. Acceso em: 20 de dic. 2013

STAKE, R. E. Investigacion con estudios de caso, 2da Edición, Ediciones Morata, Madrid, España. 1999

STOTT, Leda; RAMIL, Xosé. Metodología para el desarrollo de estudios de caso. Centro de Innovación en Tecnología para el Desarrollo Humano - itdUPM, Madrid, España. 2014. Texto disponível em: www.itd.upm.es. Acceso em. 20 dic. 2017

TORRES, Gerardo. Gobernanza de los sistemas agroalimentarios localizados. Políticas de desarrollo territorial. Rocio Rosales Ortega y Ludger Brenner (Coords) en Geografía de la gobernanza. Dinámicas multiescalares de los procesos económicoambientales, UAM-Siglo XXI Editories, México, pp. 209-230. 2015

UNIVERSIDAD DE LAS AMERICAS PUEBL. La argumentación, Universidad de las Américas Puebla. 2015

VAZQUEZ, A. Luis E.; RAMOS, G. Jesús M. Implicaciones Metodológicas en la Investigación: el caso de uma instancia de gobierno local. Atlas de Investigación Cualitativa en Ciencias Sociales, Vol. 3, pp. 558-567. CIAIQ 2019. Texto disponível em: 
https://proceedings.ciaiq.org/index.php/CIAIQ2019/article/view/2378. Acceso em 15 de feb. 2020

YACUZZI, Enrique. El estudio de caso como metodología de investigación: teoría, mecanismos causales, validación. 2013. Texto disponível em: http://www.carmaster.com.mx/MYRNA_estudiosdecaso.pdf. Acceso em:20 de nov. 2015 